Variations sur la lettre, le mètre et la mesure :

Shakespeare

\title{
Le sceau dans le théâtre de Shakespeare
}

\section{Yves Thoret}

\section{(2) OpenEdition}

1 Journals

\section{Édition électronique}

URL : http://journals.openedition.org/shakespeare/988

DOI : 10.4000/shakespeare.988

ISSN : 2271-6424

Éditeur

Société Française Shakespeare

\section{Édition imprimée}

Date de publication : 1 novembre 1996

Pagination : 195-205

Référence électronique

Yves Thoret, «Le sceau dans le théâtre de Shakespeare ", Actes des congrès de la Société française Shakespeare [En ligne], 14 | 1996, mis en ligne le 01 janvier 2007, consulté le 30 avril 2019. URL : http:// journals.openedition.org/shakespeare/988; DOI : 10.4000/shakespeare.988 


\section{Variations sur la lettre,}

le mètre et la mesure

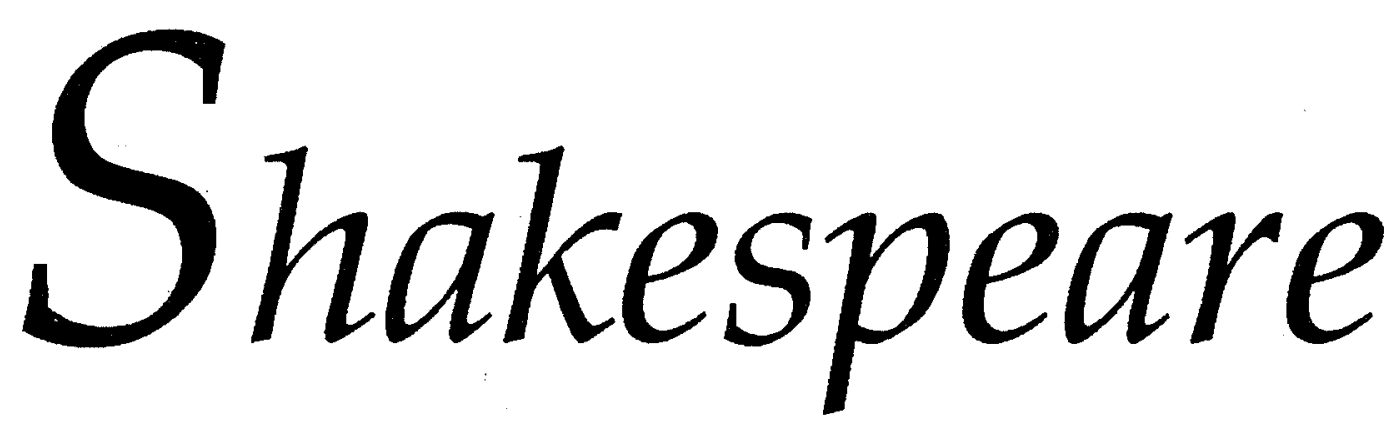

textes présentés par Dominique Goy-Blanquet

Directeur de la publication : Richard Marienstras 


\section{Le sceau dans le théâtre de Shakespeare}

On paraphe la lettre par une signature. On plie doucement la feuille sur ellemême. On place l'un sur l'autre les lacs de soie colorée qui prolongent les extrémités de la feuille. On fait couler dessus un peu de cire enflammée et on la fixe d'un coup sec avec un cachet sculpté qui imprime sur la pastille de cire la marque de l'authentique. On a apposé sur cette lettre le sceau qui convenait. Mais que représente cette marque de langage, ce label de la lettre, cet insigne solennel qui clôt la lettre sur elle-même, y appose les scellés et en interdit la lecture sauf à ouvrir en le brisant ce poing de cire fermé sur le texte ?

L'étude du théâtre de Shakespeare peut nous aider à mieux comprendre la signification symbolique du sceau. Dans la Harvard Concordance to Shakespeare, le substantif seal est cité 82 fois et le verbe to seal 42 fois. Tantôt Shakespeare désigne l'usage effectif du sceau, tantôt il représente par cette image une métaphore d'expériences aussi diverses que la naissance, l'amour, l'autorité, l'amitié, la fidélité, le sacrifice, le défi, la trahison ou la mort. Cette multiplicité de significations oblige à sélectionner certains caractères dynamiques spécifiques du sceau, que nous examinerons dans les fonctions suivantes :

a) Le sceau instaure et fixe par écrit la parole donnée

b) Il officialise un engagement d'amour

c) Il constitue une reconnaissance de dette ou un pacte

d) Il préserve le secret et authentifie l'auteur du message

e) Il peut représenter l'absorption dans le néant, une marque de mort

f) Il transmet un message d'une génération à une autre.

\section{a) Parole donnée, écrit scellé}

La principale source étymologique du mot "sceau". est le latin sigillum, diminutif de signum, signe. C'est un mot qui recouvre donc tout le champ de l'écriture. Le sceau remplaçait la signature pour les nobles qui ne savaient pas écrire, marquant l'authentification, la ratification, l'officialisation de l'auteur de la lettre et de la valeur du message. Le roi déléguait son grand Sceau au chancellier, le haut dignitaire qui avait le droit d'utiliser le sceau royal, avec l'assistance d'un garde des sceaux, plus spécialement chargé de la justice, comme de nos jours. Le sceau est un signe de pouvoir et d'autorité; il symbolise l'appartenance, l'allégeance, le lien établi avec un ordre, une autorité, une institution ou une instance.

Shakespeare insiste sur la représentation par le sceau de la parole donnée. Par exemple, Horatio rappelle que le défunt roi Hamlet affronta Fortinbras et que celui-ci perdit, avec la vie, ses terres, "en vertu d'un contrat dûment scellé, ratifié par la loi et proclamé par les héraults ${ }^{1}$." Dans Richard II, le jeune Aumerle jette son gant à celui qui vient de l'accuser de félonie : 
Tiens, voilà mon gage, le sceau de mort de ma main qui va te marquer pour l'enfer, annonçant, pour le menteur que tu es, mon épée de chevalier qui se ternira dans le sang de ton cour.

(IV.1.25-7)

Shakespeare mentionne volontiers le sceau pour rappeler le code de chevalerie dans nombre de ses œuvres, notamment les pièces historiques.

\section{b) Le sceau, symbole d'un mariage ou d'une union d'amour}

Antoine considère la main de Cléopâtre comme "un sceau royal, garant de la foi des grands cœurs" 2 . La belle Portia, dans Le Marchand de Venise, n'est pas libre de choisir elle-même parmi ses prétendants: "La volonté de la fille vivante doit se courber sous la volonté du père mort" 3 . Ce père autoritaire mais avisé impose à sa fille de n'épouser que celui qui aura réussi l'épreuve mise au point par lui-même avant de mourir, le choix des coffrets. Chacun d'eux contient un portrait ou un tableau qui présente une "morale" du choix opéré et de ses conséquences. Sans doute a-t-il pris soin de rédiger lui-même les devises gravées sur les coffrets ainsi que les billets qui, à l'intérieur, annoncent le résultat du choix. Ce protocole, analogue à un sceau, montre jusqu'à quel point ce père garde le contrôle absolu du choix de son futur gendre. C'est une parole d'outre-tombe, maintenue sous une chape de plomb, qui ne se révèle qu'à l'ouverture des coffrets, à l'issue de ces parties de loteries dont sa fille est le lot involontaire ${ }^{4}$. Jeu cruel qui empêche les perdants de courtiser à nouveau une fiancée, il entraîne dans sa logique enfouie le destin des prétendants et de la jeune héritière, cette jeune femme déjà "lasse de ce grand univers" (I.2.1-2).

Le prince du Maroc est fasciné par l'or, il pense à une pièce de monnaie portant le sceau d'un ange; Aragon se sent encouragé par l'appel à son mérite. Seul Bassanio pense au père de la jeune femme, dont la volonté s'exprime à travers ce rite matrimonial. Entrer dans une famille, c'est accepter de prendre en compte les valeurs que le père veut transmettre à sa fille et à sa lignée. Il est attiré par la beauté de Portia, ses boucles légères, son charme moqueur et grave, mais il découvre un autre rapport symbolique plus enfoui, plus refoulé, maintenant l'union entre le père mort et la fille en plein épanouissement Le sceau, comme le coffret scellé, associe la vie et la mort, la force de la vie et le pouvoir éternel de la mort :

Ainsi ces mèches d'or bouclées et serpentines

Qui font avec le vent de si jolies gambades

Sur une prétendue beauté, souvent ne sont

Qu'héritage d'une seconde tête,

Le crâne nourricier gisant dans le sépulcre....

(III.2.92-6) ${ }^{5}$. sceau :

Dans Venus and Adonis, Shakespeare symbolise l'amour et le baiser par un 
Pure lèvre, doux sceau dont ma lèvre est empreinte!

[...]

Et, ce marché conclu, (viens) mettre en la rouge cire

De ma bouche ton seing, pour ne t'en point dédire.

$$
\left(\text { v. 511-16) }{ }^{6}\right.
$$

\section{c) De la reconnaissance de dette au pacte avec le diable}

Après inventaire des allusions au sceau dans l'œuvre de Shakespeare, c'est $L e$ Marchand de Venise qui donne la représentation la plus dramatique d'un contrat scellé, la reconnaissance de dette par laquelle Antonio concède comme dédit à Shylock une livre de sa chair. Dans un article de 1923, "une névrose diabolique au dix-septième siècle ${ }^{7 "}$, Freud étudie l'histoire d'un peintre autrichien qui ne peut faire le deuil de son père et concède son âme au diable par un contrat signé avec son propre sang. Il sera délivré de cet engagement par l'intercession miraculeuse de la Vierge Marie qui fait comparaître le diable dans son sanctuaire et l'oblige à restituer le contrat au malheureux possédé. La confrontation des deux œuvres permet d'établir des correspondances entre ces personnages et ceux de la pièce, Shylock et le diable, Antonio et le peintre, Portia et la Vierge Marie, Bassanio et le père du peintre.

Il est difficile de comprendre pourquoi Shylock impose à Antonio une clause aussi rigoureuse que de gager une livre de sa chair. En laissant de côté la question des archétypes antisémites de l'époque, on observe que Shylock offre cette clause aussitôt après avoir essuyé les moqueries du marchand qui l'a comparé au diable alors qu'il citait la Bible :
Remarquez, Bassanio, Que le diable, à ses fins, peut citer l'Ecriture.
L'âme mauvaise employant le saint témoignage
Est comme un scélérat le sourire à la joue, Une pomme pourrie au ccur [...]
Oh! quels jolis dehors se donne le mensonge!

Il est permis de penser que Shylock a voulu prendre son débiteur au mot en lui proposant, "as a merry sport", un engagement démoniaque. D'ailleurs il avoue que ce morceau de viande ne lui servira qu'à appâter le poisson. De même, Freud considérait la perversion comme "les traces d'un ancien culte sexuel, célébrant Moloch ou Astarté". Freud illustrait son propos du récit d'un rituel satanique évoquant l'anthropophagie dont il examina la victime, une fillette qu'on avait obligée à manger un morceau de ses petites lèvres après une excision ${ }^{8}$.

Plusieurs personnages de la pièce (Lancelot, Solanio et Salerio) comparent Shylock au diable et Jessica insiste, quant à elle, sur l'acharnement de son père à détruire Antonio. Norman Holland rappelle qu'Otto Rank considérait Shylock comme "a money-devil"; Freud approuvait l'interprétation de Théodor Reik consi dérant Dieu et le diable comme deux figures clivées de l'imago paternelle? ${ }^{9}$ La 
clause énoncée par Portia dans le rituel du sacrifice, au moment du procès ("pas une goutte de sang ne doit être versée"), rappelle l'usage du sang pour rédiger et signer le pacte avec le diable.

En ce qui concerne le personnage d'Antonio, il présente de nombreux points communs avec le peintre qui ne trouve pas de remède à son insondable tristesse. L'explication fournie par Freud à la réaction du peintre pourrait s'appliquer tout autant à Antonio : "Voilà quelqu'un qui s'engage par écrit envers le diable pour être délivré d'une dépression affective ${ }^{10}$ ". Ce ne sont pas les trois mille ducats qui sont en jeu, mais la possibilité d'appartenir corps et biens, corps et âme (Seele, en allemand) à quelqu'un. Mieux vaut risquer ses biens, sa vie et son salut que de rester confronté à la nostalgie, à la solitude, au néant, au vide, au "rien", comme le souligne l'intéressante étude de David Willbern ${ }^{11}$. Pour Freud, le peintre signe un contrat de neuf ans avec le diable, car ce chiffre représente le nombre de mois d'une grossesse; le possédé accepte, par ce pacte, de devenir le fils du diable, pour remplacer le père qu'il a perdu. A peine sauvé des persécutions de Shylock, Antonio est prêt à engager à nouveau son corps et son âme auprès de Portia pour attester que Bassanio n'a pas failli à ses engagements en donnant son anneau de fiancailles au jeune magistrat (V.1. 249-53). Notons qu'Antonio représente les deux modèles d'homosexualité masculine décrits par Freud : il se soumet passivement au désir du père en adoptant une position féminine d'une part, et d'autre part il adopte répétitivement une attitude maternelle généreuse et protectrice envers son protégé, pouvant aller jusqu'à tout sacrifier pour lui.

Enfin, Portia intervient dans le procès pour sauver Antonio et débouter Shylock de sa plainte. Son rôle ressemble à celui de la Vierge Marie dans le cas de névrose démoniaque rapporté par Freud ou dans les histoires de miracle qui étaient souvent jouées à la Renaissance ${ }^{12}$. Notons à ce propos que le miracle décrit par Freud se produit dans le petit sanctuaire (Cella en latin) consacré à la Vierge. L'ori gine de la ville de Sceaux, proche de Paris, provient du latin Cellae: il s'y trouvait de petits celliers très appréciés car ils recélaient les bons vins des environs ${ }^{13}$. On peut donc évoquer une autre origine étymologique possible du mot "sceau" en rapport avec Cella, une loge, un sanctuaire ou une alcôve, telle celle où Portia cache ses trois coffrets derrrière un rideau.

A propos de cette pièce, il reste un point à souligner. Au moment où Shylock se prépare à sacrifier Antonio, celui-ci exprime ses dernières volontés à son ami Bassanio. Il ne se contente pas de lui rappeler son affection mais il lui demande de conter à son épouse, Portia, combien il aimait Bassanio :

Recommandez-moi à votre honorable épouse,

Racontez-lui comment a fini Antonio,

Dites combien je vous aimai, rendez justice au mort;

Et, quand tout sera dit, faites-la juge

Si Bassanio n'a pas été chéri......

(IV. 1. 270-74). 
Ce dernier vœu mérite d'être étudié. Il ne révèle pas simplement un possible attachement homosexuel entre Antonio et son ami mais il exprime le désir pour Antonio de réunir dans son souvenir le couple formé par Portia et Bassanio; on peut trouver la même configuration dans le miracle décrit par Freud, où le diable peut restituer le pacte à sa victime quand il se trouve soumis à l'influence de la figure maternelle bienfaisante, la Vierge Marie. Le désir qui s'exprime là n'est pas celui d'une jalousie œdipienne mais, au contraire, le vœu exprimé par l'enfant de rétablir l'union affective et sexuelle entre les parents, de restaurer leur commerce et leur échange affectif, dont l'enfant serait en quelque sorte, à la fois le parrain et le produit.

Ce modèle fournit une explication au comportement mystérieux d'Antonio; inconsolable d'avoir perdu ses parents, exerçant contre ses goûts d'artiste la profession de marchand, il intervient à plusieurs reprises pour favoriser, restaurer ou renforcer l'union entre Portia et Bassanio. C'est cet objectif fantasmatique, et lui seul, qui peut redonner corps au désir de vie d'Antonio. On peut interpréter ainsi le dernier "miracle" de la pièce : Portia remet à Antonio une lettre scellée lui annonçant l'entrée triomphale de ses galions dans le port de Venise ${ }^{14}$. Sans risquer de se faire taxer de surinterprétation, on peut penser que cette image représente la satisfaction de l'enfant lorsqu'il est assuré de l'existence de liens d'amour intenses entre les parents. Portia précisera que cette lettre est arrivée entre ses mains par "chance", mais elle n'en dira pas plus sur son origine ni sur le sceau qui la maintient fermée. Ce caractère énigmatique semble manifester la correspondance avec un désir infantile très enfoui.

\section{d) Le sceau authentifie l'auteur du message et garde son contenu secret}

C'est Hamlet qui illustre le mieux cette fonction. Voguant vers l'Angleterre, Hamlet ne trouve pas le sommeil. Oubliant toute prudence, cédant aux impulsions inspirées par la divinité qui protège sa destinée et lui fournit un cadre, il se couvre d'un caban, se glisse dans le réduit où sont les bagages de ses compagnons et $\mathrm{y}$ trouve la lettre officielle que Claudius a remise, après l'avoir scellée, à Rosencrantz et Guildenstern. Il découvre que Claudius ordonne aux Anglais de l'exécuter sans délai.

Horatio se refusant à croire le récit de Hamlet, celui-ci lui remet, en présence du public, la lettre en question. La preuve existe maintenant que le roi a ordonné sa mort. Le prince pouvait encore douter des confidences du Spectre, il pouvait encore s'interroger sur les réactions de Claudius au spectacle donné par les comédiens; mais il ne peut plus douter des intentions criminelles du roi depuis qu'il a trouvé cette lettre et brisé le sceau qui la maintenait secrète.

De retour au Danemark, Hamlet raconte à Horatio comment il a découvert ce complot. André Green souligne qu'à ce moment-là, Hamlet repensait à la manipulation qu'il avait lui-même ourdie lorsqu'il avait ajouté de sa main quelques phrases assassines au prologue de la pièce que les comédiens devaient jouer devant la cour ${ }^{15}$. L'expression utilisée par Shakespeare, "pendant que j'en étais encore à expliquer le prologue à mon cerveau, les autres étaient déjà entrés au cœur de la pièce" (V.2.30-1), permet à l'auteur de s'exprimer là, en montrant l'emballement de 
l'intrigue, en avance sur sa propre création; Shakespeare lui-même parle au public par la bouche de Hamlet sur ce point ${ }^{16}$.

En violant le secret de cette correspondance, Hamlet découvre que ses adversaires n'en sont plus au prologue de la pièce mais qu'ils ont pris de l'avance sur lui . Ils ont entamé l'action principale, qui doit aboutir à sa mort. Il se sent enveloppé par leur malveillance comme dans un filet et réagit aussitôt par une contreattaque. Il contrefait la lettre officielle en imitant les formules ampoulées du langage diplomatique et donne à ce faux l'apparence du vrai, en y apposant le sceau de son père, qu'il avait conservé sur lui. Les deux sceaux représentent les deux images du père pour Hamlet. Celui de Claudius est celui de son rival :

\section{Celui qui a tué mon roi, prostitué ma mère, Qui s'est jeté entre mes droits et la couronne, Qui a lancé sa nasse sur ma vie...}

$$
\text { (V.2.64-6). }
$$

Le sceau du vieux roi est celui de la légitimité et la figure divine qui protège et donne forme à ses ambitions. C'est le sceau de la filiation, de l'identification enfin réussie de Hamlet à son père, même si ce mouvement leur donne à tous deux une apparence un peu diaphane de "goblin". Ce sceau appose sur une fausse lettre la marque de l'autorité la plus authentique. Hamlet a retrouvé son père et se place dans la lignée. Hamlet a dissipé ainsi toute idée de substitution d'enfant (changeling) en opérant cette falsification de lettres avec le seul sceau qui soit authentique pour lui, celui de son père, Hamlet, roi de Danemark.

Le père de Hamlet se sert de lui comme d'une plume et cette filiation rétablie marque l'ouverture du combat final qui s'est engagé à outrance entre Hamlet et Claudius : "The interim is mine". Hamlet vient de sceller son destin avec le signet de son père. Dans cette scène, le vrai et le faux s'entrecroisent en permancence dans ce moment de vérité où, selon l'expression de Jacques Lacan, "le mensonge apparaît comme garant de la vérité dans laquelle il subsiste ${ }^{17 "}$.

Citons rapidement trois autres pièces dans lesquelles le sceau authentifie l'auteur d'un message.

- La Nuit des rois, où Malvolio croit reconnaître, grâce à son sceau, les lettres d'amour de la comtesse, alors qu'elles ont été contrefaites par Maria.

- Le Conte d'hiver, où un protocole solennel régit l'ouverture des sceaux qui protègent le message d'Apollon. L'oracle innocente Hermione mais Léontès n'y voit que fausseté. Son impudence cèdera lorsqu'on lui annoncera la mort de son fils puis d'Hermione. Il fera sceller sous une même sépulture le souvenir de ces deux êtres et y fera graver le texte de ses méfaits, comme s'il voulait ainsi restaurer le texte de l'oracle. Pauline brisera le sceau de cette culpabilité en redonnant vie à la statue de la reine et en permettant au temps et à la vie de reprendre leur cours.

- Mesure pour mesure, où le duc, déguisé en moine, livre au prévôt un secret d'Etat en lui faisant reconnaître l'écriture et le sceau du duc lui-même. Dès qu'il a reconnu l'auteur du message, et dès qu'il a reçu confidence du contenu de la lettre, 
le prévôt devient l'allié du duc; l'authentification de l'auteur du message entraine une modification importante de la position subjective du destinataire de la lettre. Shakespeare compare le caractère rassurant de cette révélation à l'apparition d'une étoile ("the unfolding star") qui annonce l'aube et autorise le berger à laisser son troupeau se disperser, de même que le duc encourage le prévôt à déplier fébrilement sa lettre (IV. 2).

On ne peut mieux représenter ce que Jacques Lacan appelle l'instance de la lettre, "le support matériel que le discours concret emprunte au langage". La lettre se situe entre l'écrit et la parole, elle est une instance qui agit "dans la transformation dramatique que le dialogue peut opérer dans le sujet ${ }^{18 "}$.

\section{e) Le sceau, marque de mort}

Shakespeare utilise aussi la métaphore du sceau pour représenter la mort et l'entrée dans le néant. Dans Henri $V$, Exeter raconte au roi la mort héroïque au combat du valeureux York. Blessé grièvement, ce soldat a recommandé ses services à son souverain, a demandé à son compagnon mort de l'attendre pour gagner le ciel, l'a baisé aux lèvres et "ainsi, marié à la mort, il scella de son sang le testament de cette noble finn." Le témoin de cette scène décrit alors ses propres réactions :

Ce beau et doux spectacle m'a arraché des pleurs que j'aurais voulu retenir; mais ma fermeté d'homme étant à bout, ma mère tout entière a surgi à mes yeux et m'a fait fondre en larmes.

$$
\text { (IV.6.26-32) }{ }^{19} \text {. }
$$

Ce récit fait apparaître successivement l'imago paternelle, la noblesse, la fidélité, la loyauté de ce capitaine, puis l'imago maternelle, l'immense compassion qui tente de compenser le passage au néant et à l'intemporalité.

Le sceau peut aussi représenter le viol. Ce crime, le plus souvent, ne peut se dire. Les soudards, "abusent les vierges sur les sceaux brisés du parjure" (Henri V, IV.1.164). Dans Le Viol de Lucrèce, l'héroïne est envahie par le chagrin, que seul peut atténuer le souffle des mots, la lettre qu'elle adresse à son seigneur, en y apposant son sceau et en ajoutant : "Hâte-toi, hâte-toi plus encore" [With more than haste] (v. 1331). La fille de Titus Andronicus, Lavinia, a été mutilée après le viol et ne peut parler. Elle ne s'exprime que par le recours au langage écrit, la lecture et l'écriture. Elle choisit le passage d'Ovide où est décrit le viol de Philomèle et inscrit avec un bâton sur le sol le nom de ses agresseurs. Ses frères s'empresseront de recopier ces noms sur des tablettes pour fixer leur serment de vengeance.

Le sceau peut représenter la nature même du criminel. Ainsi Richard III est décrit comme "evil-mark'd, seal'd in nativity", monstre marqué au mal dès sa naissance, né pour faire le mal. (I.3.228). C'est cette marque de tyrannie chez Gloucester que Richard Marienstras a analysé de façon approfondie ${ }^{20}$.

Le sceau peut aussi symboliser le suicide. Tantôt le héros motive son geste par la disparition de l'être aimé. Ainsi Antoine n'a plus rien qui le retienne à la vie quand on lui annonce la mort de Cléopâtre : 
Puisque la torche est éteinte, couchons-nous sans plus tarder. [...]

Apposons notre sceau, et tout est fini.

(IV.14.40-9)

Cléopâtre, accusée par Antoine d'avoir un cour de glace, appelle sur elle, sur son fils et l'Egypte les fureurs de l'Apocalypse :

Que de mon cour glacé le ciel engendre une grêle empoisonnée à sa source! [...]. Que les autres grêlons abolissent la mémoire de mon ventre [the memory of my womb] et que tous mes braves Egyptiens gisent sans sépulture, abandonnés aux mouches et aux moustiques.....

(III.13.159-67)

Dans Roméo et Juliette, la jeune femme disparaît avec la même discrétion résignée qu'Antoine, tandis que Roméo évoque, au moment de se suicider, comme Cléopâtre, la matrice maternelle :

Scellez, mes lèvres, portes du souffle, d'un baiser auquel j'ai droit, un marché hors du temps avec la mort qui enveloppe tout, qui aspire tout, qui engrosse tout [a dateless bargain to engrossing deathl.

(V.3.112-15)

\section{- f) Le sceau, garant de la transmission}

Les exemples cités utilisent le sceau pour représenter le chaos, le néant, l'éntrée sans retour dans le royaume des morts. Dans ses dernières pièces, Shakespeare transcende l'antinomie entre l'amour et la mort. Cléopâtre rappelle que "la nature envie, pour créer, l'étoffe inépuisable du rêve" (V.2.97-8). Elle retrouve le contact avec Antoine au moment où elle place son sein entre les mâchoires de l'aspic, comme entre les branches d'une pince à sceller. Au moment de se frapper, Antoine lui-même rêvait de "gambades joyeuses sur une couche de fleurs... ${ }^{21 "}$.

Le critique $J$. W. Lever a montré le développement de la création shakespearienne entre le poème intitulé Vénus et Adonis, (où Vénus échouait à - séduire Adonis qui mourait peu après, blessé par un sanglier), et les dernières pièces, notamment Antoine et Cléopâtre, dans lesquelles Shakespeare offre à ses héros la possibilité de se réunir à nouveau, au-delà des portes de la mort, dans un monde imaginaire. Shakespeare décrit alors une variété de paradis où se restaure l'union de l'amour et de la mort. "En consommant fièvreusement cette union, les mortels amoureux deviennent immortels : non pas dans un monde naturel, ni dans un paradis spirituel, mais dans un autre champ, le domaine inconnu de l'imagination [...]. Vénus et Adonis, après leur chute, se relèveront comme Cléopâtre et Antoine pour entrer triomphalement dans le royaume de la seconde chance ${ }^{22}$ ". 
Le sceau correspond à ces métamorphoses qui s'opérent sur ce bloc-notes magique où nos sentiments font apparaître, en encre sympathique, des mots qui ne deviennent visibles qu'au contact des larmes.

"Nous gambaderons joyeux sur une couche de fleurs...."

Antoine s'élance vers la mort comme vers une fiancée. Cléopâtre se pare pour sa dernière scène des ornements de la Reine de l'Apocalypse. Au dernier jour, seul l'agneau, symbole de l'amour, pourra lever le septième sceau du Livre.

Shakespeare porte ses personnages au-delà des limites de la mort, dans le domaine imaginaire de la seconde chance. Cette représentation mythique nous aide à reconnaître la mission testamentaire du sceau, fonction plus générale déjà illustrée par le jeune Marc Antoine dans une pièce antérieure. Après l'assassinat de Jules César, il serrait en gage d'amitié la main sanglante des conjurés, puis présentait le corps sacrifié au peuple de Rome, brandissant le testament scellé où César avait inscrit ses dernières volontés: "Here is the will ! and under Cæsar's seal" (Jules César, III.2.242). C'est la seconde chance de César, de retrouver par cet écrit qu'Antoine hésite à décacheter, la faveur des Romains. Le sceau permet de rétablir la continuité entre les générations, de supporter le caractère éphémère de la vie et de rétablir le fondement de l'ordre symbolique, la lignée.

Les premières fonctions du sceau visaient à authentifier, à fixer, à instaurer une relation. Autre fonction du scellement, la représentation de l'origine et de la mort, de la création et de la destruction. Enfin, il est apparu que les imagos paternelles et maternelles se condensaient pour conférer au sceau une fonction de relais, de transmission, pour toujours laisser vivre l'espoir d'une seconde chance. Et si, suggérait un participant au Congrès, la psychanalyse comme le théâtre permettait d'accorder à ses personnages une seconde chance?

Yves THORET Université de Paris-X

1 Hamlet, I.1.86-9. Sauf indication contraire, les citations de Shakespeare sont données dans ma propre traduction.

2 Antoine et Cléopâtre, III.2.125, trad. André Gide, La Pléiade, Paris, 1977.

3 I.2.23, traduction François-Victor Hugo, La Pléiade.

4 Y. Thoret, La Théâtralité, étude freudienne, Paris, Dunod, 1993.

$5 \quad$ Sigmund Freud, "Le motif du choix des coffrets" (1913), dans L'Inquiétante Etrangeté et autres essais, Paris, P.U.F., 1985, pp. 61-81. Sur ce thème, voir aussi 


\section{LE SCEAU}

l'article de Joseph Wagner, "In the name of the dead father", Literature and Psychology, Proceedings of the 12th annual conference, Lisbonne, I.S.P.A., 1996. Cette citation du Marchand de Venise et les suivantes sont traduites par J. Grosjean, Paris, Flammarion, 1994.

6 Traduction Jean Fuzier, La Pléiade.

7 Freud, dans L'Inquiétante Etrangeté, pp. 265-315.

8 Lettre de Freud à W. Fliess du 24 janv. 1897, dans Briefe an Wilhelm Fliess 1887-1904, Frankfurt am Main, S. Fisher Verlag, 1986, pp. 239-41; cette anecdote ne figure pas dans la traduction française, La Naissance de la psychanalyse, Paris, P.U.F., 1973, pp. 166-68.

9 Travaux cités par Norman Holland, Psychoanalysis and Shakespeare, New York \& Londres, McGraw-Hill, 1966, p. 232.

10 "Une névrose diabolique", op. cit. p. 281. Voir aussi L. Stephens, " 'A wilderness of monkeys' : a psychodynamic study of The Merchant of Venice" in The Undiscover'd Country, New essays on psychoanalysis and Shakespeare, ed. B. J. Sokol, Londres, Free Association Books, 1993, pp. 91-129.

11 "Shakespeare's Nothing", in Representing Shakespeare, ed. M. Schwarz et C. Kahn, Baltimore \& Londres, The Johns Hopkins U. P., 1980, pp. 244-63. Les principaux travaux de David Willbern sont rassemblés dans son ouvrage Poetic Will : Shakespeare and the Play of Language, U. of Pennsylvania Press, 1996.

12 Molly M. Mahood, Introduction to The Merchant of Venice, The New Cambridge Shakespeare, C. U.P., 1987, pp. 9 et 24.

13 Je dois cette précision à Madame Elisabeth Manivel, angliciste, qui a vérifié que la première mention du nom de la ville de Sceaux figure dans les archives du Prieuré de Longpont, en 1120.

14 "Unseal this letter soon;

There you shall find three of your argosies

Are richly come to harbour suddenly.

You shall not know by what strange accident

I chanced on this letter". (V. 1. 275-79).

15 Hamlet et Hamlet, Paris, Balland, 1982, pp. 175-77. 
16 Voir traduction Yves Bonnefoy: "Avant que je n'aie pu la lui expliquer, / Mon cerveau commençait la pièce", V.2.30-1, Folio Gallimard, 1988

17 "L'instance de la lettre dans l'inconscient ou la raison depuis Freud", Ecrits, Paris, Seuil, 1966, pp. $495-528$ (p. 524).

$18 \quad$ Ibid., pp. 495 et 503.

19 Traduction F.-V. Hugo, La Pléiade.

20 "D'un corps monstrueux", dans Le Tyran : Shakespeare contre Richard III, ed. D. Goy-Blanquet et R. Marienstras, Amiens, STERNE, 1990, pp. 1-26.

21 Voir la traduction de F.-V. Hugo,"là où les âmes couchent sur des fleurs nous irons main dans la main et nous éblouirons les esprits de notre auguste apparition", IV.14.51-2, Garnier-Flammarion, Paris, 1965.

22 "Venus and the second chance", Shakespeare Survey 15, 1962, pp. 81-8, travaux dont Marie-Thérèse Jones-Davies souligne l'intérêt dans Shakespeare, le théâtre du monde, Paris, Balland, 1987, pp. 94-5. 